\title{
Rapamycin reverses insulin resistance (IR) in high-glucose medium without causing IR in normoglycemic medium
}

\author{
OV Leontieva ${ }^{*, 1}$, ZN Demidenko ${ }^{1}$ and MV Blagosklonny ${ }^{*, 1}$
}

Mammalian target of rapamycin (mTOR) is involved in insulin resistance (IR) and diabetic retinopathy. In retinal pigment epithelial (RPE) cells, insulin activates the mTOR pathway, inducing hypoxia-inducible factor-1 $\alpha$ (HIF-1 $\alpha$ ) and HIF-dependent transcription in serum-free minimum essential medium Eagle (MEM). Serendipitously, we found that insulin failed to induce the HIF-1 $\alpha$-dependent response, when RPE cells were cultured in Dulbecco's modification of Eagle's medium (DMEM). Whereas concentration of glucose in MEM corresponds to normal glucose levels in blood (5.5 mM), its concentration in DMEM corresponds to severe diabetic hyperglycemia $(25 \mathrm{mM})$. Addition of glucose to MEM also caused IR. Glucose-mediated IR was characterized by basal activation of mTORC1 and its poor inducibility by insulin. Basal levels of phosphorylated S6 kinase (S6K), S6 and insulin receptor substrate 1 (IRS1) S635/639 were high, whereas their inducibilities were decreased. Insulin-induced Akt phosphorylation was decreased and restored by rapamycin and an inhibitor of S6K. IR was associated with de-phosphorylation of IRS1 at S1011, which was reversed by rapamycin. Both short (16-40 h) and chronic (2 weeks) treatment with rapamycin reversed IR. Furthermore, rapamycin did not impair Akt activation in RPE cells cultured in normoglycemic media. In contrast, Torin 1 blocked Akt activation by insulin. We conclude that by activating mTOR/S6K glucose causes feedback IR, preventable by rapamycin. Rapamycin does not cause IR in RPE cells regardless of the duration of treatment. We confirmed that rapamycin also did not impair phosphorylation of Akt at T308 and S473 in normal myoblast C2C12 cells. Our work provides insights in glucoseinduced IR and suggests therapeutic approaches to treat patients with IR and severe hyperglycemia and to prevent diabetic complications such as retinopathy. Also our results prompt to reconsider physiological relevance of numerous data and paradigms on IR given that most cell lines are cultured with grossly super-physiological levels of glucose.

Cell Death and Disease (2014) 5, e1214; doi:10.1038/cddis.2014.178; published online 8 May 2014

Subject Category: Experimental Medicine

Insulin resistance (IR) is the most common pathological condition associated with age- and obesity-related diseases, including type II diabetes, atherosclerosis, hypertension, neurodegeneration and cancer. ${ }^{1}$ Furthermore, IR is associated with aging in diverse species from humans to Drosophila $^{2,3}$ and Caenorhabditis elegans. ${ }^{4}$ One of the explanations of this universal link is that both IR and aging involve target of rapamycin. ${ }^{5}$ Mammalian target of rapamycin (mTOR) is activated by numerous signals including insulin and glucose. ${ }^{6,7}$ In turn, mTOR increases cellular metabolism, protein synthesis, cellular growth ${ }^{7-9}$ and cellular senescence. $^{10-19}$ Insulin initiates activation of insulin receptor substrate (IRS), AKT and mTOR and its target S6 kinase (S6K; Figure 1), which in turn phosphorylates IRS, thus limiting insulin signaling. ${ }^{20-25}$ This feedback loop can be activated by signals that stimulate mTOR/S6K, including nutrients, growth factors, hormones, oxygen, Ras and oncogenic kinases. ${ }^{20-25}$ Overstimulation of mTOR leads not only to IR but also to hypoxia-inducible factor- $1 \alpha$ (HIF-1 $\alpha$ ) expression and cellular senescence. ${ }^{26}$ Both strong insulin signaling and IR can be associated with overactivation of mTOR, which is a negative factor in aging and its diseases. ${ }^{27}$ The complex relationship was previously discussed in detail. $^{27}$

Previously, we described a simple cell model to measure insulin response in retinal pigment epithelial (RPE) cells transfected with HRE-Luc (HRE, HIF-responsive element). ${ }^{28}$ Insulin activates mTOR, which increases synthesis of HIF-1, which in turn induces HRE-Luc. This process is completely blocked by rapamycin, indicating that the signal transduction from insulin to HIF-1 solely depends on mTORC $1 .{ }^{28} \mathrm{HIF}-1$ may foster pathology including retinopathy, therefore, rapamycin can prevent retinopathy. $5,29,30$

Accidentally, we found that, while robustly inducing HRELuc in minimum essential medium Eagle (MEM), insulin did not cause any significant response in RPE cells cultured in Dulbecco's modification of Eagle's medium (DMEM). DMEM and MEM media differ in glucose concentration: in MEM levels

\footnotetext{
${ }^{1}$ Department of Cell Stress Biology, Roswell Park Cancer Institute, Elm and Carlton Streets, Buffalo, NY, USA

*Corresponding authors: OV Leontieva or MV Blagosklonny, Department of Cell Stress Biology, Roswell Park Cancer Institute, Elm and Carlton Streets, Buffalo, NY 14263, USA. Tel: + 1716 8458086, Fax: + 1716 8453944; Email: Olga.leontieva@ roswellpark.org or Blagosklonny@oncotarget.com

Keywords: insulin; Akt; mTOR; S6K; rapalogs

Abbreviations: mTOR, mammalian target of rapamycin; ERK, extracellular signal-regulated kinase; HIF, hypoxia-inducible factor; HRE-Luc, HIF-responsive elementluciferase; S6K, S6 kinase; IRS, insulin receptor substrate; IR, insulin resistance; DMEM, Dulbecco's modification of Eagle's medium; MEM, minimum essential medium Eagle; RPE, retinal pigment epithelial

Received 17.2.14; revised 17.3.14; accepted 26.3.14; Edited by G Melino
} 


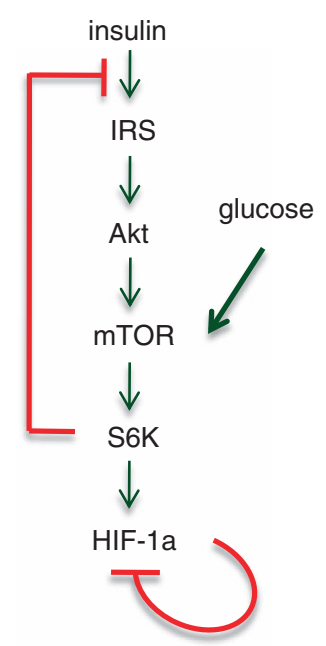

Figure 1 The insulin/mTOR/HIF-1 pathway: a simplified schema. Glucose activates mTOR, which in turn blocks insulin signaling (a feedback loop). The mTOR pathway increases translation of the HIF-1 $\alpha$ RNA. However, accumulation of the HIF- $1 \alpha$ protein is tightly limited by its degradation via a PHD-feedback loop under normoxia. ${ }^{28,66}$ Red lines, inhibitory feedback loops

of glucose correspond to normal physiological levels in human blood $(100 \mathrm{mg} / \mathrm{dl}$ or $5.5 \mathrm{mM})$, whereas in DMEM levels of glucose are five times higher, corresponding to untreated severe diabetes. This accidental observation led to important implications for the mechanism of IR, treatment of diabetes and the use of anti-aging agents such as rapamycin to prevent diseases. Although phenomenon of the loss of insulin response in DMEM seems trivial, we investigated the phenomenon in depth.

\section{Results}

Glucose-induced IR. In RPE cells, insulin activates mTOR (Figure 1), which in turn increases translation of the HIF-1 $\alpha$ mRNA, transiently induces HIF- $1 \alpha$ and the HIF- $1 \alpha$-dependent transcriptional response. ${ }^{28}$ As shown in Figure $2 \mathrm{a}$, both insulin and serum induced the HIF- $1 \alpha$-dependent response in serum-free MEM medium. In contrast, in DMEM, this response is induced by serum but not by insulin (Figure 2a). Next, we investigated whether the difference in response to insulin in MEM versus DMEM could be due to the difference in glucose concentrations. We cultured RPE cells with increasing concentrations of glucose added to MEM. Increasing concentration of glucose gradually decreased the responsiveness of cells to insulin (Figure 2b). Given that rapamycin also blocked HIF-1 $\alpha$ dependent response, ${ }^{28}$ we next checked whether mTORC1 is inhibited in DMEM medium.

High basal phospho-S6 (pS6) and poor pS6 inducibility. To determine the mTORC1 activity, we measured levels of pS6, a downstream target of mTORC1/S6K, in DMEM and MEM (Figure 3a). In MEM, levels of phospho-S6 (especially at $\mathrm{S} 235 / 236$ ) were low and insulin dramatically induced $\mathrm{S} 6$ phosphorylation. In DMEM, basal levels of pS6 were high and insulin only marginally increased pS6. Thus, high basal levels of pS6 and blunted insulin response were associated with glucose-induced IR. As a positive control for IR, cells were cultured with high dose of insulin overnight, so that such cells could not respond to acute stimulation with insulin. This insulin-induced IR is a positive control for complete IR, which was accompanied by highly phosphorylated S6 (Figure 3a) and did not further rerespond to insulin.

Restoration of AKT signaling by rapamycin in DMEMinduced IR. Next, we investigated the activation of Akt by insulin. Akt is an upstream activator of mTOR. In MEM, basal levels of phospho-AKT(T308) and phospho-AKT(S473) were undetectable and insulin dramatically induced AKT phosphorylation on both sites (Figure 3). In the positive control for IR (insulin-induced IR), induction of p-Akt-473 was impaired, whereas p-Akt-308 was not induced at all. In DMEM, induction of p-Akt, especially at T308 was impaired too. Pre-treatment with rapamycin (either overnight or for 2 weeks) completely restored insulin-induced AKT phosphorylation in DMEM medium. Thus, insulin-induced AKT phosphorylation was reduced in glucose-induced IR and was restored by rapamycin. Importantly, both overnight and chronic treatment with rapamycin restored response of AKT to insulin.

Alterations in IRS1 phosphorylation. The phosphorylation pattern of IRS1 in response to insulin was also changed in IR. Using antibodies against phospho-S1101, we found lack of phosphorylation of this residue in DMEM- and insulininduced IR (Figure 3a). In DMEM, rapamycin restored phosphorylation of IRS1 at S1101. Rapamycin prevented the mobility shift of IRS1 in response to insulin, indicating block of phosphorylation of some other sites. One of such sites is 5636/639 (Figure 3b). Although the mechanism of S1101 dephosphorylation in IR is unclear, the absence of phospho-S1101 can be used as a marker of IR, which is reversed by rapamycin. Rapamycin exerted a dose-response effect on p-IRS1 (S1101). Rapamycin restored basal level of S1101phosphorylation but prevented the mobility shift of IRS-1 in response to insulin stimulation (Figure 4). Furthermore, this effect was also detectable at as low as $0.1 \mathrm{nM}$ rapamycin, which did not inhibit pS6 (Figure 4). Thus, at very low doses that affected neither phosphorylation of S6K nor phosphorylation of S6 and AKT, rapamycin detectably affected phosphorylation of IRS1 S1101.

Phospho-IRS1 S636/639 in IR. Changes in phosphorylation of IRS1 at S1101 could serve as a marker of IR, which is reversed by rapamycin. However, the mechanistic significance of this phenomenon is not clear. We therefore next investigated phosphorylation of IRS1 on S636/639, which was reported to be phosphorylated in the S6K1 feedback loop. Like S6, IRS1-S636/639 was not phosphorylated in MEM (Figure 3b). In contrast, its level of phosphorylation was increased in DMEM, whereas its inducibility by insulin was weak (Figure $3 b$ ). In fact, in positive control (insulin-induced IR), there was no response (even paradoxical decrease of S636/639 phosphorylation in response to insulin). Rapamycin decreased basal level of p-IRS1 S636/639 in DMEM (Figure 3b). Thus, phosphorylation of IRS1 at S636/639 mirrored the phosphorylation state of S6, suggesting that this IRS1 site is a substrate of 


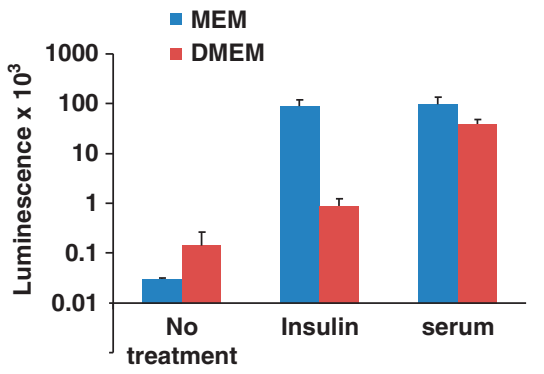

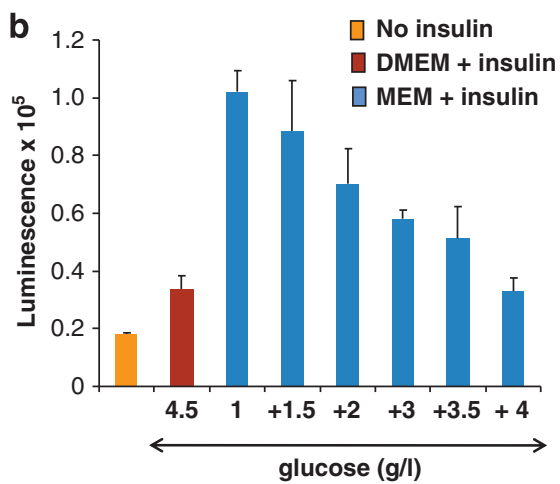

Figure 2 Induction of HRE-Luc in MEM, DMEM and MEM + additional glucose. RPE cells was plated in either MEM or DMEM with FBS. After cell attachment, the medium was changed to serum-free MEM or DMEM, as indicated. Cells were transfected with HRE-Luc (HIF-responsive-luciferase construct). After 1 day, cells were treated with $1 \mu \mathrm{g} / \mathrm{ml}$ insulin and $10 \%$ FBS as indicated. After $16 \mathrm{~h}$, cellular luciferase activity was measured. (a) Comparison of MEM and DMEM. (b) Increasing concentrations of glucose (g/l) were added to MEM medium
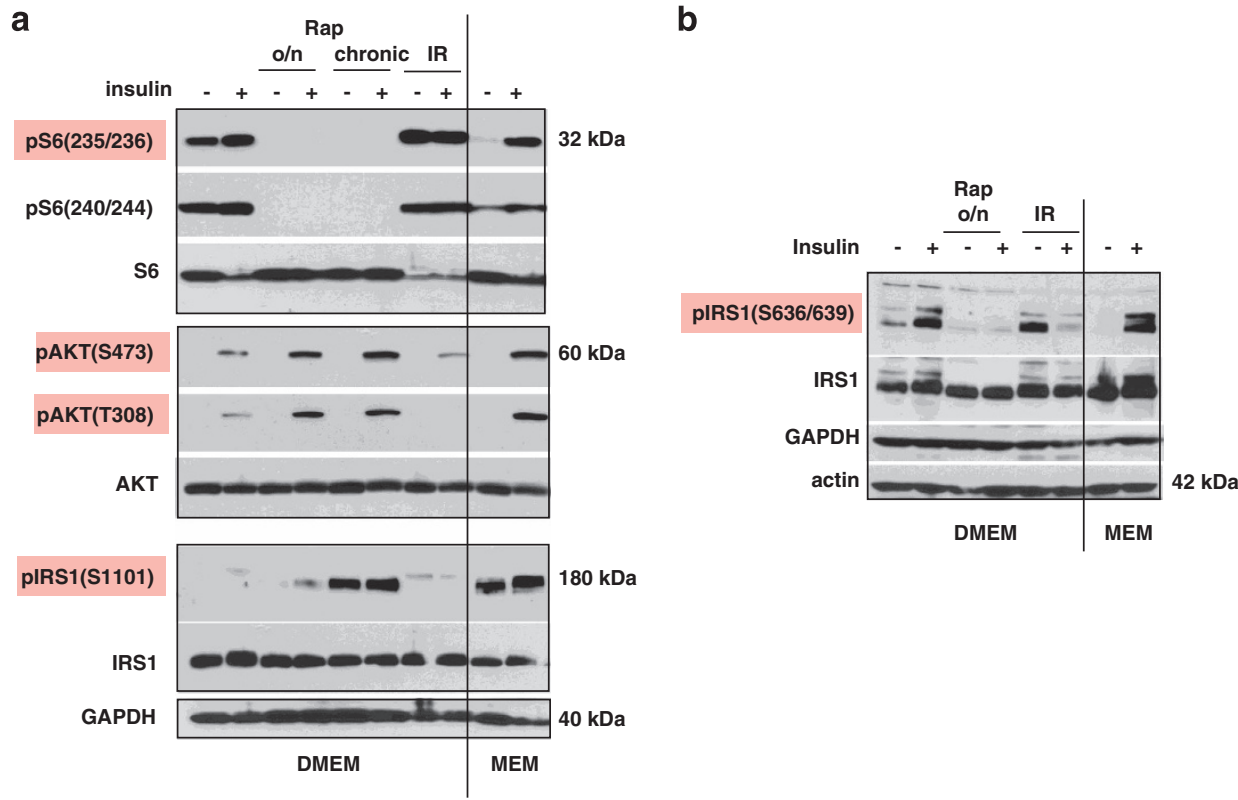

Figure 3 Effects of rapamycin on insulin resistance in DMEM. Immunoblot analysis. (a) RPE cells were maintained in either DMEM (25 mM glucose) or MEM (5.5 mM glucose). Cells were incubated in serum-free medium for $42-44 \mathrm{~h}$ and then were stimulated with $1 \mu \mathrm{g} / \mathrm{ml}$ insulin for 15 min and lysed. If indicated Rap, stimulation in the presence of rapamycin. Rap, $10 \mathrm{nM}$ rapamycin; o/n, rapamycin overnight before stimulation with insulin; chronic, rapamycin for 2 weeks before stimulation; IR, cells were treated with $1 \mu \mathrm{g} / \mathrm{ml}$ insulin overnight before stimulation; (b) RPE cell whole-cell lysates as in (a) were rerun in part and immunoblotting was performed with the indicated antibodies

S6K. As expected, rapamycin not only blocked basal level of p-IRS1 S636/639 but also prevented its phosphorylation in response to insulin.

S6K inhibitor restores Akt activation. PF-4708671, an inhibitor of $\mathrm{S} 6 \mathrm{~K},{ }^{31}$ inhibited basal level of phospho-S6 (Figure 4). Unlike rapamycin, it did not block completely an increase in pS6 in response to insulin. Importantly, PF4708671 restored pAKT induction in response to insulin. PF4708671 also decreased basal level of p-IRS1 S636/639 but could not prevent phosphorylation of IRS1 in response to insulin (Supplementary Figure 1). These data are in agreement with the effect of PF-4708671 on S6 phosphorylation (Figure 4), indicating that PF-4708671 more potently inhibits basal level than inducible S6K activity.
Rapamycin does not cause IR. As shown in Figures 3 and 4, rapamycin reversed hyperglycemia-induced IR. Next, we examined whether rapamycin may still cause IR in normoglycemia. As expected, insulin caused phosphorylation of AKT and S6 (Figure 5a). Although blocking phosphorylation of the targets downstream of mTORC1 (such as p-S6, p-IRS1-S636/639), rapamycin did not affect phosphorylation of AKT at either S473 or T308. We next tested the effect of Torin 1, a double inhibitor of mTORC1 and mTORC2. Like rapamyicn, it blocked downstream targets of mTORC1, phospho-S6K and phospho-S6. Unlike rapamycin, which did not affect insulin-induced AKT phosphorylation, Torin 1 abolished phosphorylation of AKT by insulin. These effects are consistent with inhibition of mTORC2 by Torin 1. We conclude that both acute and chronic (a 2-week) treatment 


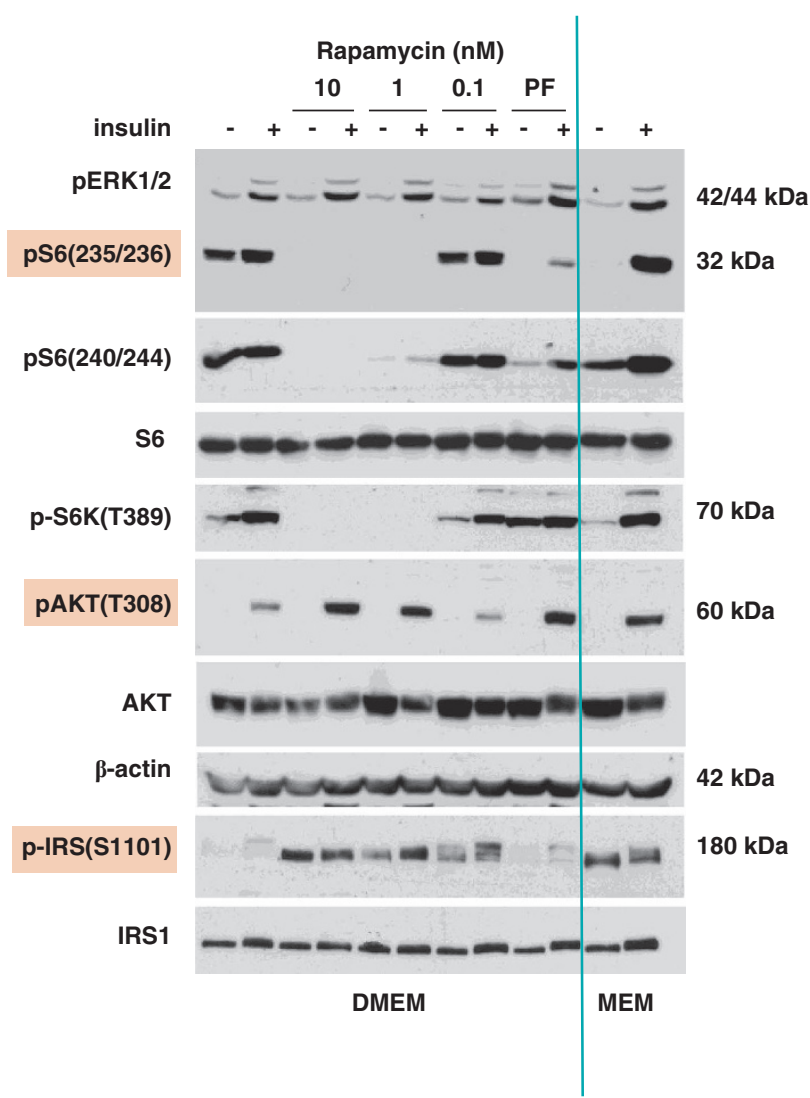

Figure 4 Effects of S6K inhibitor and dose-response to rapamycin. Immunoblot analysis. RPE cells were maintained in either DMEM ( $25 \mathrm{mM}$ glucose) or MEM ( $5.5 \mathrm{mM}$ glucose) complete medium. Cells were incubated in serum-free respective medium in the absence or presence of rapamycin as indicated (in $\mathrm{nM}$ ) or $10 \mu \mathrm{M}$ PF4708671 (inhibitor of S6K1 kinase) for 42-44 $\mathrm{h}$ and then were stimulated with $1 \mu \mathrm{g} / \mathrm{ml}$ insulin for $20 \mathrm{~min}$ and lysed. Immunoblotting of membranes from two separate gels

with rapamycin neither inhibited $\mathrm{mTORC2}$ nor caused IR in RPE cells.

Rapamycin does not cause IR in immortalized mouse myoblast $\mathrm{C} 2 \mathrm{C} 12$ cells. $\mathrm{C} 2 \mathrm{C} 12$ cells are often used to study IR. In normoglycemic serum-free medium, insulin induced phospho-AKT and phospho-S6 (Figure 6). Rapamycin (acute and chronic) did not affect phosphorylation of AKT at T308. Furthermore, $4 \mathrm{~h}$ pre-treatment with rapamycin even increased insulin-induced phospho-AKT S473. Torin 1 also inhibited phospho-S6. It also inhibited phosphorylation of AKT at S473, especially at later time point (46h). Pretreatment with Torin 1 also abrogated the mobility shift of total AKT caused by insulin. We conclude that rapamycin did not cause Torin-like impairment of AKT activation. In C2C12 cells, IRS1 phosphorylation at S1101 was not affected by rapamycin and Torin 1, although both agents decreased the mobility shift caused by insulin. This mobility shift depends on feedback loop via mTOR in both RPE and C2C12 cells.

\section{Discussion}

Here we found that hyperglycemic medium caused IR in RPE cells. IR was associated with phosphorylation of the S6K and its substrates, S6 and IRS1 (S636/639). Chronic activation of mTOR (by high glucose) results in diminished activation of AKT in response to insulin. Even a lesser effect of insulin was detected downstream of mTOR, including a complete loss of the HIF- $1 \alpha$-dependent response. Thus, basal activation of mTOR precludes the inducibility of its downstream targets. Rapamycin restored phosphorylation of AKT at Threonine 308 and Serine 473 in response to insulin stimulation. At the same time, rapamycin blocks activation of the targets downstream of mTOR (Figure 7), thus preventing the negative consequences such as induction of pseudo-hypoxic state, cellular senescence and signal resistance. ${ }^{26}$ The inhibitor of S6K reproduced effects of rapamycin on insulin-induced Akt activation, albeit it did not completely prevent insulin-inducible activation of S6K. We also addressed the controversial issue of rapamycin-induced IR. Increased mTOR activity causes IR in cell culture and in animals, including humans, reversible by rapamycin and calorie restriction, which both inhibit mTOR. ${ }^{21,32-42}$ However, it was reported that chronic rapamycin treatment can cause either IR or glucose intolerance in some conditions. ${ }^{43-48}$ Such metabolic alterations strikingly resemble 'starvation diabetes' developed during starvation or severe caloric restriction. ${ }^{49}$ Not co-incidentally, rapamycin is caloric restriction-mimetic, causing a beneficial condition named 'beneficial pseudo-diabetes' .

We demonstrated that acute and chronic rapamycin treatment did not impair AKT phosphorylation in two cell lines. In contrast, Torin 1 blocked AKT S473 phosphorylation in both cell lines and AKT T308 phosphorylation in RPE cells. In both cell lines, the effects of rapamycin did not mirror the effects of Torin 1, suggesting that rapamycin did not inhibit mTORC2, at least drastically. However, a modest decrease in phospho-Akt levels in $\mathrm{C} 2 \mathrm{C} 12$ cells was previously reported. ${ }^{50}$

As IR was also manifested by the lack of induction of targets downstream of mTORC1 in response to insulin (such as pS6, HIF $1 \alpha$ and expression of HRE-Luc), and the same events were prevented by rapamycin, one could conclude that rapamycin might cause IR. However, this lack of insulintriggered induction of mTOR-dependent targets in the presence of rapamycin is accompanied by the improvement of the upstream insulin signaling (Figure $7 \mathrm{~b}$ ). This condition could be defined as pseudo-resistance.

The effect of insulin, hyperglycemia and rapamycin on the phosphorylation of IRS1 is extremely complex. Various kinases, such as S6K1, mTOR, Akt, PKC, Erk1/2, p90Rsk, each phosphorylate its numerous (more than 50 serine/ threonine residues) phosphorylation sites. ${ }^{51}$ Not only kinases are redundant and overlapping, they are cell type- and condition-specific. ${ }^{41,51-58}$ Without attempting to clarify the mechanisms, we found that basal levels of phospho-IRS1 S1101 were decreased in IR cells. p-IRS1 S1101 levels were restored by rapamycin, which also prevented the mobility shift of IRS1 induced by insulin. One of the explanations could be that rapamycin prevented a feedback phosphorylation of IRS1, exemplified by S636/639 phosphorylation. The same effect was caused by PF-4708671 and Torin 1. We suggest that a feedback phosphorylation of IRS1 on several sites may be blocked by rapamycin. Yet this did not decrease but even increased AKT activation and ERK phosphorylation by insulin. It is important to emphasize that knockdown of IRS1 in mice 
a

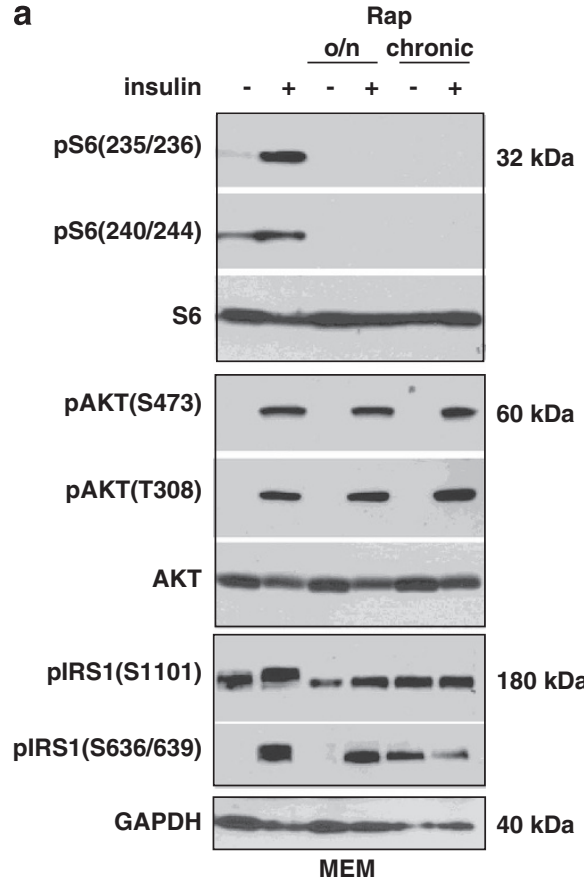

b

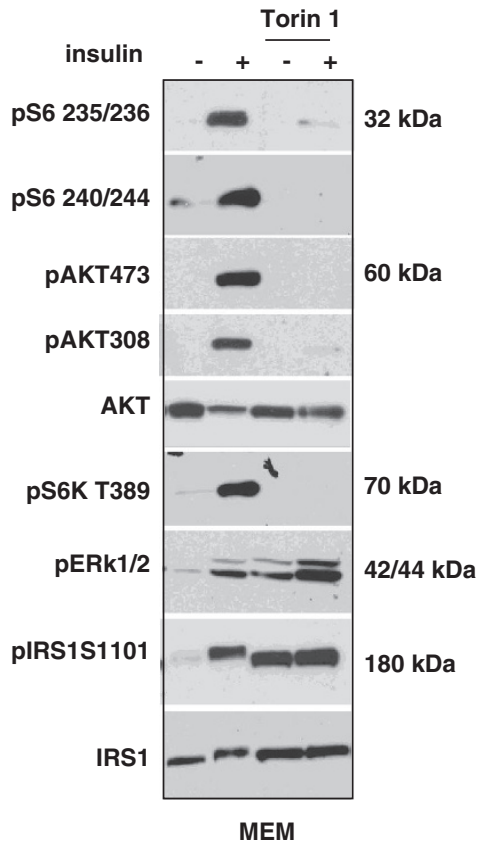

Figure 5 Comparison of rapamycin and Torin 1 in RPE cells. Immunoblot analysis. RPE cells were incubated in serum-free MEM medium (42-44 h) and pre-treated as indicated with either $10 \mathrm{nM}$ rapamycin (a) or $300 \mathrm{nM}$ torin 1 for $42-44 \mathrm{~h}$ (b) and then were stimulated with $1 \mu \mathrm{g} / \mathrm{ml}$ insulin for 15 min and lysed. o/n, rapamycin overnight before stimulation; chronic, rapamycin for 2 weeks before stimulation. Immunoblotting of membranes from two separate gels

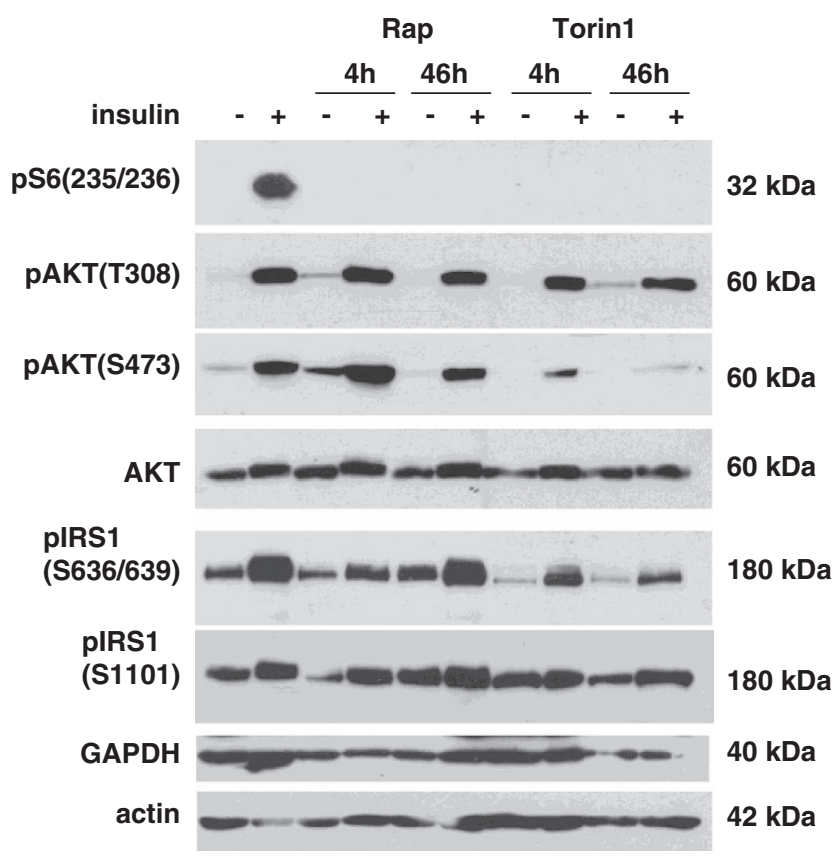

Figure 6 Comparison of rapamycin and Torin 1 in $\mathrm{C} 2 \mathrm{C} 12$ cells. Immunoblot analysis. $\mathrm{C} 2 \mathrm{C} 12$ cells were incubated in serum-free low-glucose $(5.5 \mathrm{mM}) \mathrm{DMEM}$ for $\sim 46 \mathrm{~h}$ and then stimulated with $1 \mu \mathrm{g} / \mathrm{ml}$ insulin for $15 \mathrm{~min}$ and lysed. If indicated, cells were treated with $100 \mathrm{nM}$ rapamycin or $300 \mathrm{nM}$ Torin 1 for either $46 \mathrm{~h}$ or $4 \mathrm{~h}$ before stimulation with insulin. Blots were produced from immunoblotting of membranes from two separate gels

improves health and extends lifespan in mice. ${ }^{59}$ This supports the notion that neither the status of IRS 1 per se nor IR actually determines whether the effect on health is negative or positive. Instead, it is the activity of mTOR that matters as was previously discussed in detail. ${ }^{27}$ We can summarize that rapamycin reverses, not causes, IR in cell culture. This occurs even during chronic administration of rapamycin. In fact, the improvement of metabolic parameters was observed in chronic use of rapamycin in mice. ${ }^{60,61}$ Given that rapamycin blocks the events downstream of mTOR, pseudo-resistance could be diagnosed (if such parameters are measured), however, such pseudo-resistance must have beneficial health effects. For example, suppression of cellular aging, reduction of HIF- $1 \alpha$ and VEGF may prevent diabetic retinopathy. In fact, rapamycin prevents retinopathy in animal models. ${ }^{5,29,30}$

Second, hyperglycemia causes IR. ${ }^{62,63}$ In diabetic patients, this can be an obstacle, requiring high doses of insulin. Highdose insulin therapy is associated with side effects as well as insulin-induced IR. ${ }^{64}$ We suggest that pretreatment of such patients with oral rapamycin will reverse IR. Then lower doses of insulin would be effective in decreasing glucose levels. This would avoid hypoglycemia or the danger of insulin overdosing. Also pretreatment with rapamycin will abrogate harmful activation of mTOR, whereas increasing the upstream effects of insulin. Another aspect is that the S6K inhibitor PF-4708671 reproduced many effects of rapamycin on reversing IR. S6K inhibitor potently blocked basal pS6 but did not completely block inducible pS6. Thus, its effect would be milder and more specific, given that S6K has less targets than mTOR does.

\section{Materials and Methods}

Cell lines and reagents. RPE, also known as ARPE-19, and immortalized mouse myoblast cell line, C2C12, were obtained from ATCC (Manassas, VA, USA). RPE cells were maintained in either MEM or high-glucose DMEM, both media without sodium pyruvate, plus $10 \%$ FBS and $\mathrm{C} 2 \mathrm{C} 12$ cells were cultured in 


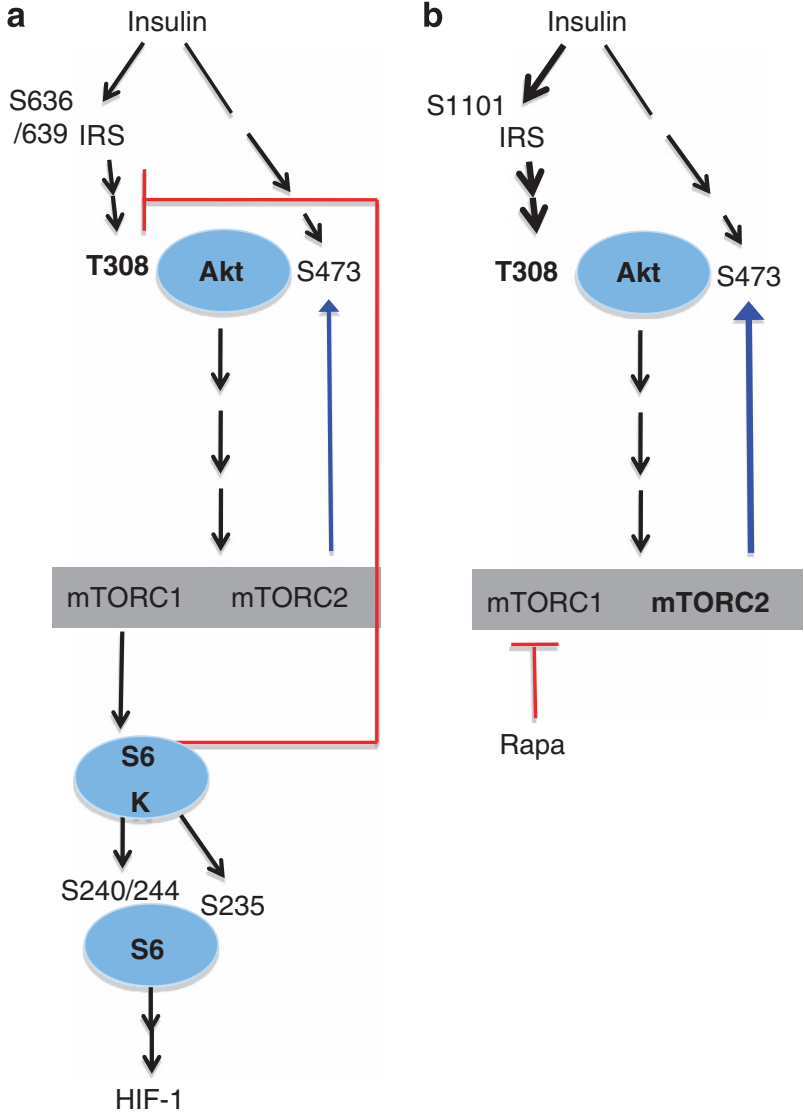

Figure 7 The insulin/mTOR pathway in RPE cells. (a) Insulin activates IRS and causes Akt phosphorylation, resulting in mTOR activation. As a negative feedback loop, mTOR and S6K both block insulin signaling. In high-glucose conditions, S6K is chronically active, causing insulin resistance. This resistance is characterized (in RPE cells) by phospho-S636/639 IRS1, p-S6 and impaired insulin-induced activation of Akt. (b) In the presence of rapamycin, the events downstream of mTORC1 are blocked and therefore there is no feedback inhibition of insulin signaling

high-glucose DMEM without pyruvate plus 10\% FBS. Serum-free low-glucose DMEM was used in some experiments. Rapamycin was obtained from LC Laboratories (Woburn, MA, USA). Torin 1 was purchased from Selleckchem (Houston, TX, USA). Insulin (as $10 \mathrm{mg} / \mathrm{ml}$ solution) and PF 4708671 were obtained from Sigma-Aldrich (St. Louis, MO, USA). Stock solutions were prepared in DMSO: rapamycin at $5 \mathrm{mM}$ and $50 \mu \mathrm{M}$; Torin 1 at $3 \mathrm{mM}$; PF4708671 at $10 \mathrm{mM}$.

Immunoblot analysis. Immunoblotting was performed as described previously. ${ }^{31}$ In brief, whole-cell lysates were prepared using boiling lysis buffer ( $1 \% \mathrm{SDS}, 10 \mathrm{mM}$ Tris $\cdot \mathrm{HCl}, \mathrm{pH}$ 7.4). Equal amounts of proteins were separated using Criterion or mini gradient polyacrylamide gels (Bio-Rad, Hercules, CA, USA) and transferred to PVDF membranes. The following rabbit antibodies for: phospho-S6 (S235/236), phospho-S6 (S240/244), phospho ERK $1 / 2$, phospho-Thr 389 p70S6K, phospho-AKT (S473) and phospho-AKT (T308), phospho-IRS1 (S1101), phospho-IRS1(S636/639), IRS1 and mouse anti-S6 antibody were from Cell Signaling Biotechnology (Danvers, MA, USA). Rabbit anti-actin and mouse monoclonal anti-GAPDH antibodies were from Sigma-Aldrich and Invitrogen (Camarillo, CA, USA), respectively. Secondary anti-rabbit and anti-mouse HRPconjugated antibodies were from Cell Signaling Biotechnology.

Transient transfection and plasmids. The HIF-responsive HRE-Luc plasmid was used previously. ${ }^{65}$ HRE-Luc was obtained by subcloning three copies of a double-stranded 21-bp oligonucleotide (5'-AGTGACTACGTG CTGCCT-3') in the pGL3 promoter vector (Promega, Madison, WI, USA), digested with Kpnl and Mlul. For the analysis of the expression of HRE-Luc,
50000 cells were plated in 24-well plates either in DMEM or in MEM with $10 \%$ FBS. Then medium was changed for serum-free DMEM or MEM, respectively, and then transfected with plasmids by using TransFast transfection reagent (Promega) according to the manufacturer's recommendations. After $2 \mathrm{~h}$ of incubation with the plasmid-lipid suspension, the medium was changed and cells were cultured for an additional day. Then cells were treated with either $1 \mu \mathrm{g} / \mathrm{ml}$ insulin or $10 \% \mathrm{FBS}$, if indicated. After $16 \mathrm{~h}$, cells were lysed and analyzed for luciferase activity as described previously. ${ }^{65}$

\section{Conflict of Interest \\ The authors declare no conflict of interest}

Acknowledgements. This work was supported in part by Roswell Park Cancer Institute, Buffalo.

1. Barzilai N, Huffman DM, Muzumdar RH, Bartke A. The critical role of metabolic pathways in aging. Diabetes 2012; 61: 1315-1322.

2. Haselton A, Sharmin E, Schrader J, Sah M, Poon P, Fridell YW. Partial ablation of adult Drosophila insulin-producing neurons modulates glucose homeostasis and extends life span without insulin resistance. Cell Cycle 2010; 9: 3063-3071.

3. Luong N, Davies CR, Wessells RJ, Graham SM, King MT, Veech R et al. Activated FOXO-mediated insulin resistance is blocked by reduction of TOR activity. Cell Metab 2006; 4: 133-142.

4. Rahman MM, Stuchlick O, El-Karim EG, Stuart R, Kipreos ET, Wells L. Intracellular protein glycosylation modulates insulin mediated lifespan in C. elegans. Aging (Albany NY) 2010; 2: 678-690.

5. Blagosklonny MV. TOR-centric view on insulin resistance and diabetic complications: perspective for endocrinologists and gerontologists. Cell Death Dis 2013; 4: e964.

6. Wullschleger S, Loewith R, Hall MN. TOR signaling in growth and metabolism. Cell 2006; 124: $471-484$.

7. Sengupta S, Peterson TR, Sabatini DM. Regulation of the mTOR complex 1 pathway by nutrients, growth factors, and stress. Mol Cell 2010; 40: 310-322.

8. Duran RV, Hall MN. Glutaminolysis feeds mTORC1. Cell Cycle 2012; 11: 4107-4108.

9. Cornu M, Albert V, Hall MN. mTOR in aging, metabolism, and cancer. Curr Opin Genet Dev 2013; 23: 53-62.

10. Demidenko ZN, Blagosklonny MV. Growth stimulation leads to cellular senescence when the cell cycle is blocked. Cell Cycle 2008; 7: 3355-3361.

11. Blagosklonny MV, Hall MN. Growth and aging: a common molecular mechanism. Aging (Albany NY) 2009; 1: 357-362.

12. Kolesnichenko M, Hong L, Liao R, Vogt PK, Sun P. Attenuation of TORC1 signaling delays replicative and oncogenic RAS-induced senescence. Cell Cycle 2012; 11: 2391-2401.

13. Hinojosa CA, Mgbemena V, Van Roekel S, Austad SN, Miller RA, Bose S et al. Entericdelivered rapamycin enhances resistance of aged mice to pneumococcal pneumonia through reduced cellular senescence. Exp Gerontol 2012; 47: 958-965.

14. Demidenko ZN, Korotchkina LG, Gudkov AV, Blagosklonny MV. Paradoxical suppression of cellular senescence by p53. Proc Natl Acad Sci USA 2010; 107: 9660-9664.

15. Leontieva OV, Natarajan V, Demidenko ZN, Burdelya LG, Gudkov AV, Blagosklonny MV. Hypoxia suppresses conversion from proliferative arrest to cellular senescence. Proc Natl Acad Sci USA 2012; 109: 13314-13318.

16. Leontieva OV, Lenzo F, Demidenko ZN, Blagosklonny MV. Hyper-mitogenic drive coexists with mitotic incompetence in senescent cells. Cell Cycle 2012; 11: 4642-4649.

17. Menendez JA, Vellon L, Oliveras-Ferraros C, Cufi S, Vazquez-Martin A. mTOR-regulated senescence and autophagy during reprogramming of somatic cells to pluripotency: a roadmap from energy metabolism to stem cell renewal and aging. Cell Cycle 2011; 10: 3658-3677.

18. Iglesias-Bartolome R, Patel V, Cotrim A, Leelahavanichkul K, Molinolo AA, Mitchell JB et al. mTOR inhibition prevents epithelial stem cell senescence and protects from radiationinduced mucositis. Cell Stem Cell 2012; 11: 401-414.

19. Chen C, Liu Y, Zheng P. mTOR regulation and therapeutic rejuvenation of aging hematopoietic stem cells. Sci Signal 2009; 2: ra75.

20. Um SH, Frigerio F, Watanabe M, Picard F, Joaquin M, Sticker M et al. Absence of S6K1 protects against age- and diet-induced obesity while enhancing insulin sensitivity. Nature 2004; 431: 200-205.

21. Tremblay F, Marette A. Amino acid and insulin signaling via the mTOR/p70 S6 kinase pathway. A negative feedback mechanism leading to insulin resistance in skeletal muscle cells. J Biol Chem 2001; 276: 38052-38060.

22. Harrington LS, Findlay GM, Lamb RF. Restraining PI3K: mTOR signalling goes back to the membrane. Trends Biochem Sci 2005; 30: 35-42.

23. Corradetti MN, Guan KL. Upstream of the mammalian target of rapamycin: do all roads pass through mTOR? Oncogene 2006; 25: 6347-6360. 
24. Yu Y, Yoon SO, Poulogiannis G, Yang Q, Ma XM, Villen J et al. Phosphoproteomic analysis identifies Grb10 as an mTORC1 substrate that negatively regulates insulin signaling Science 2011; 332: 1322-1326.

25. Hsu PP, Kang SA, Rameseder J, Zhang Y, Ottina KA, Lim D et al. The mTOR-regulated phosphoproteome reveals a mechanism of mTORC1-mediated inhibition of growth factor signaling. Science 2011; 332: 1317-1322.

26. Leontieva OV, Blagosklonny MV. M(o)TOR of pseudo-hypoxic state in aging: Rapamycin to the rescue. Cell Cycle 2014; 13: 509-515

27. Blagosklonny MV. Once again on rapamycin-induced insulin resistance and longevity: despite of or owing to. Aging (Albany NY) 2012; 4: 350-358.

28. Demidenko ZN, Blagosklonny MV. The purpose of the HIF-1/PHD feedback loop: to limit mTOR-induced HIF-1alpha. Cell Cycle 2011; 10: 1557-1562.

29. Kolosova NG, Muraleva NA, Zhdankina AA, Stefanova NA, Fursova AZ, Blagosklonny MV Prevention of age-related macular degeneration-like retinopathy by rapamycin in rats. $A m J$ Pathol 2012; 181: 472-477.

30. Zheng XF. Chemoprevention of age-related macular regeneration (AMD) with rapamycin Aging (Albany NY) 2012; 4: 375-376.

31. Pearce LR, Alton GR, Richter DT, Kath JC, Lingardo L, Chapman J et al. Characterization of PF-4708671, a novel and highly specific inhibitor of p70 ribosomal S6 kinase (S6K1) Biochem J 2010; 431: 245-255.

32. Einstein FH, Fishman S, Bauman J, Thompson RF, Huffman DM, Atzmon G et al. Enhanced activation of a 'nutrient-sensing' pathway with age contributes to insulin resistance. FASEB J 2008; 22: 3450-3457.

33. Khamzina L, Veilleux A, Bergeron S, Marette A. Increased activation of the mammalian target of rapamycin pathway in liver and skeletal muscle of obese rats: possible involvement in obesity-linked insulin resistance. Endocrinology 2005; 146: 1473-1481

34. Mordier S, lynedjian PB. Activation of mammalian target of rapamycin complex 1 and insulin resistance induced by palmitate in hepatocytes. Biochem Biophys Res Commun 2007; 362: 206-211

35. Saha AK, Xu XJ, Balon TW, Brandon A, Kraegen EW, Ruderman NB. Insulin resistance due to nutrient excess: is it a consequence of AMPK downregulation? Cell Cycle 2011; 10: 3447-3451.

36. Shah OJ, Wang Z, Hunter T. Inappropriate activation of the TSC/Rheb/mTOR/S6K cassette induces IRS $1 / 2$ depletion, insulin resistance, and cell survival deficiencies. Curr Biol 2004; 14: 1650-1656.

37. Tremblay F, Krebs M, Dombrowski L, Brehm A, Bernroider E, Roth E et al. Overactivation of $\mathrm{S} 6$ kinase 1 as a cause of human insulin resistance during increased amino acid availability. Diabetes 2005; 54: 2674-2684.

38. Krebs M, Brunmair B, Brehm A, Artwohl M, Szendroedi J, Nowotny P et al. The Mammalian target of rapamycin pathway regulates nutrient-sensitive glucose uptake in man. Diabetes 2007: 56: 1600-1607.

39. Shaw RJ, Lamia KA, Vasquez D, Koo SH, Bardeesy N, Depinho RA et al. The kinase LKB1 mediates glucose homeostasis in liver and therapeutic effects of metformin. Science 2005; 310: $1642-1646$.

40. He G, Sung YM, Digiovanni J, Fischer SM. Thiazolidinediones inhibit insulin-like growth factor-i-induced activation of p70S6 kinase and suppress insulin-like growth factor-I tumorpromoting activity. Cancer Res 2006; 66: 1873-1878.

41. Tzatsos A, Kandror KV. Nutrients suppress phosphatidylinositol 3-kinase/Akt signaling via raptor-dependent mTOR-mediated insulin receptor substrate 1 phosphorylation. Mol Cell Biol. 2006; 26: 63-76.

42. Vodenik B, Rovira J, Campistol JM. Mammalian target of rapamycin and diabetes: what does the current evidence tell us? Transplant Proc 2009; 41: S31-S38.

43. Leibowitz G, Cerasi E, Ketzinel-Gilad M. The role of $m T O R$ in the adaptation and failure of beta-cells in type 2 diabetes. Diabetes Obes Metab 2008; 10(Suppl 4): 157-169.

44. Shigeyama Y, Kobayashi T, Kido Y, Hashimoto N, Asahara S, Matsuda T et al. Biphasic response of pancreatic beta-cell mass to ablation of tuberous sclerosis complex 2 in mice. Mol Cell Biol 2008; 28: 2971-2979.

45. Chang GR, Wu YY, Chiu YS, Chen WY, Liao JW, Hsu HM et al. Long-term administration of rapamycin reduces adiposity, but impairs glucose tolerance in high-fat diet-fed KK/HIJ mice. Basic Clin Pharmacol Toxicol 2009; 105: 188-198.

46. Lamming DW, Ye L, Katajisto P, Goncalves MD, Saitoh M, Stevens DM et al. Rapamycininduced insulin resistance is mediated by mTORC2 loss and uncoupled from longevity. Science 2012; 335: 1638-1643.

47. Lamming DW, Ye L, Astle CM, Baur JA, Sabatini DM, Harrison DE. Young and old genetically heterogeneous HET3 mice on a rapamycin diet are glucose intolerant but insulin sensitive. Aging Cell 2013; 12: 712-718.
48. Ye L, Widlund AL, Sims CA, Lamming DW, Guan Y, Davis JG et al. Rapamycin doses sufficient to extend lifespan do not compromise muscle mitochondrial content or endurance. Aging (Albany NY) 2013; 5: 539-550.

49. Blagosklonny MV. Rapamycin-induced glucose intolerance: Hunger or starvation diabetes. Cell Cycle 2011; 10: 4217-4224.

50. Ye L, Varamini B, Lamming DW, Sabatini DM, Baur JA. Rapamycin has a biphasic effect on insulin sensitivity in $\mathrm{C} 2 \mathrm{C} 12$ myotubes due to sequential disruption of mTORC1 and mTORC2. Front Genet 2012; 3: 177.

51. Copps KD, White MF. Regulation of insulin sensitivity by serine/threonine phosphorylation of insulin receptor substrate proteins IRS1 and IRS2. Diabetologia 2012; 55: 2562-2582.

52. Ueno M, Carvalheira JB, Tambascia RC, Bezerra RM, Amaral ME, Carneiro EM et al. Regulation of insulin signalling by hyperinsulinaemia: role of IRS-1/2 serine phosphorylation and the mTOR/p70 S6K pathway. Diabetologia 2005; 48: 506-518.

53. Glynn EL, Lujan HL, Kramer VJ, Drummond MJ, DiCarlo SE, Rasmussen BB. A chronic increase in physical activity inhibits fed-state mTOR/S6K1 signaling and reduces IRS-1 serine phosphorylation in rat skeletal muscle. Appl Physiol Nutr Metab 2008; 33: 93-101.

54. Zhang J, Gao Z, Yin J, Quon MJ, Ye J. S6K directly phosphorylates IRS-1 on Ser-270 to promote insulin resistance in response to TNF-(alpha) signaling through IKK2. J Biol Chem 2008; 283: 35375-35382.

55. Rajan M, Fagerholm S, Jšnsson C, Kjølhede $P$, Turkina M, Strålfors $P$. Phosphorylation of IRS1 at serine 307 in response to insulin in human adipocytes is not likely to be catalyzed by p70 ribosomal S6 kinase. PLoS One 2013; 8: e59725.

56. Smadja-Lamère N1, Shum M, Déléris $P$, Roux PP, Abe J, Marette A. Insulin activates RSK (p90 ribosomal S6 kinase) to trigger a new negative feedback loop that regulates insulin signaling for glucose metabolism. J Biol Chem 2013; 288: 31165-31176.

57. Tanti JF, Jager J. Cellular mechanisms of insulin resistance: role of stress-regulated serine kinases and insulin receptor substrates (IRS) serine phosphorylation. Curr Opin Pharmacol 2009; 9: 753-762.

58. Nyman E, Fagerholm S, Jullesson D, StrCElfors P, Cedersund G. Mechanistic explanations for counter-intuitive phosphorylation dynamics of the insulin receptor and insulin receptor substrate-1 in response to insulin in murine adipocytes. FEBS J 2012; 279: 987-999.

59. Selman C, Lingard S, Choudhury Al, Batterham RL, Claret M, Clements M et al. Evidence for lifespan extension and delayed age-related biomarkers in insulin receptor substrate 1 null mice. FASEB J 2008; 22: 807-818.

60. Fang Y, Bartke A. Prolonged rapamycin treatment led to beneficial metabolic switch. Aging (Albany NY) 2013; 5: 328-329.

61. Leontieva OV, Paszkiewicz G, Demidenko ZN, Blagosklonny MV. Resveratrol potentiates rapamycin to prevent hyperinsulinemia and obesity in male mice on high fat diet. Cell Death Dis 2013; 4: e472.

62. Vuorinen-Markkola H, Koivisto VA, Yki-Jarvinen H. Mechanisms of hyperglycemia-induced insulin resistance in whole body and skeletal muscle of type I diabetic patients. Diabetes 1992; 41: 571-580.

63. Yki-Jarvinen $\mathrm{H}$. Acute and chronic effects of hyperglycaemia on glucose metabolism: implications for the development of new therapies. Diabet Med 1997; 14(Suppl 3): S32-S37.

64. Okamoto MM, Anhe GF, Sabino-Silva R, Marques MF, Freitas HS, Mori RC et al. Intensive insulin treatment induces insulin resistance in diabetic rats by impairing glucose metabolism-related mechanisms in muscle and liver. J Endocrinol 2011; 211: 55-64.

65. Salnikow K, Kluz T, Costa M, Piquemal D, Demidenko ZN, Xie K et al. The regulation of hypoxic genes by calcium involves c-Jun/AP-1, which cooperates with hypoxia-inducible factor 1 in response to hypoxia. Mol Cell Biol 2002; 22: 1734-1741.

66. Menendez JA, Alarcón T, Joven J. Gerometabolites: the pseudohypoxic aging side of cancer oncometabolites. Cell Cycle 2014; 13: 699-709.

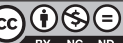

Cell Death and Disease is an open-access journal published by Nature Publishing Group. This work is licensed under a Creative Commons Attribution-NonCommercialNoDerivs 3.0 Unported License. The images or other third party material in this article are included in the article's Creative Commons license, unless indicated otherwise in the credit line; if the material is not included under the Creative Commons license, users will need to obtain permission from the license holder to reproduce the material. To view a copy of this license, visit http://creativecommons.org/licenses/ by-nc-nd/3.0/

Supplementary Information accompanies this paper on Cell Death and Disease website (http://www.nature.com/cddis) 